\title{
A role for mixed lineage kinases in granule cell apoptosis induced by cytoskeletal disruption
}

\author{
Georg Johannes Müller, ${ }^{*}, 1$ Marie Aavang Geist, ${ }^{\dagger}, 1$ Lone Merete Veng, $†$ Mette Georgi Willesen, $\dagger$ \\ Flemming Fryd Johansen,* Marcel Leist† and Elisabetta Vaudano $† \dagger$ \\ * Molecular Neuropathology Group, University of Copenhagen, Denmark \\ $\dagger$ H. Lundbeck A/S, Valby, Denmark \\ $\ddagger E N K A M$ Pharmaceuticals A/S, Copenhagen, Denmark
}

\begin{abstract}
Microtubule disruption by colchicine induces apoptosis in selected neuronal populations. However, little is known about the upstream death signalling events mediating the neurotoxicity. We investigated first whether colchicine-induced granule cell apoptosis activates the c-Jun $\mathrm{N}$-terminal kinase (JNK) pathway. Cultured murine cerebellar granule cells were exposed to $1 \mu \mathrm{m}$ colchicine for $24 \mathrm{~h}$. Activation of the JNK pathway was detected by western blotting as well as immunocytochemistry using antibodies against phosphoc-Jun (p-c-Jun). Next, adult male rats were injected intracerebroventricularly with colchicine $(10 \mu \mathrm{g})$, and JNK pathway activation in dentate granule cells (DGCs) was detected by antibodies against p-c-Jun. The second part of the study tested the involvement of mixed lineage kinases (MLK) as
\end{abstract}

Cytoskeletal alterations and impaired axonal transport followed by progressive neuronal loss are important features of several neurodegenerative diseases. In particular, cytoskeletal disruption has been linked to neurodegeneration in Alzheimer's disease (AD) and other tauopathies (Spillantini and Goedert 1998), and recent data suggest a possible role also in the pathogenesis of multiple sclerosis (Brück and Stadelmann 2003). Dysfunction and/or destabilization of microtubules leads to impaired intracellular trafficking and accumulation of mitochondria and peroxisomes, which in turn may cause synaptic loss and increased axonal excitotoxicity (Mandelkow et al. 2003). In addition, perturbed axonal transport prevents trafficking of neurotrophic molecules. Blockade of axonal transport by inhibition of the microtubule assembly can thus mimic the cytoskeletal alterations of $\mathrm{AD}$ in animal models.

Colchicine is an alkaloid derivative, which binds irreversibly to microtubules, thereby causing depolymeri- upstream activators of the JNK pathway in colchicine toxicity, using CEP-1347, a potent MLK inhibitor. In vitro, significant inhibition of the JNK pathway, activated by colchicine, was achieved by 100-300 nM CEP-1347, which blocked both activation of cell death proteases and apoptosis. Moreover, CEP-1347 markedly delayed neurite fragmentation and cell degeneration. In vivo, CEP-1347 (1 mg/kg) significantly prevented $\mathrm{p}$-c-jun increase following injection of colchicine, and enhanced survival of DGCs. We conclude that colchicineinduced neuronal apoptosis involves the JNK/MLK pathway, and that protection of granule cells can be achieved by MLK inhibition.

Keywords: CEP-1347, colchicine, mixed-lineage-kinase, c-Jun N-terminal kinase, granule cells.

zation and preventing their assembly. Thus, colchicine potently inhibits axoplasmic transport and mitosis (Hanson and Edstrom 1978). In the rat, intracerebroventricular colchicine injections cause cytoskeletal damage leading to

Received September 29, 2005; revised manuscript received October 11, 2005; accepted October 12, 2005.

Address correspondence and reprint requests to Marcel Leist, H. Lundbeck A/S, Ottiliavej 9, 2500 Valby, Denmark.

E-mail: male@lundbeck.com

${ }^{1}$ These authors contributed equally to this study.

Abbreviations used: AD, Alzheimer's disease; AEBSF, 4-(2-Aminoethyl) benzene sulfonyl fluoride hydrochloride; CGCs, cerebellar granule cells; DG, dentate gyrus; DGCs, dentate granule cells; DIV, days in vitro; $\mathrm{H} / \mathrm{E}$, hematoxylin/eosin; ICC, immunocytochemistry; IHC, immunohistochemistry; JNK, c-Jun N-terminal kinase; MAPKKK, mitogenactivated protein kinase kinase kinases; MKK, mitogen-activated protein kinase kinase; MLKs, mixed lineage kinases; MTT, 3-(4,5-demethylthiazole-2-yl)-2,5-diphenyltetrasodium bromide; p-c-Jun, phosphoc-Jun; s.c., subcutaneously. 
death of cerebellar granule cells (CGCs), olfactory bulb neurons, cells of the subventricular zone as well as dentate granule cells (DGCs) and basal forebrain cholinergic neurons (Goldschmidt and Steward 1982). The mechanisms underlying selective vulnerability to colchicine toxicity remain unknown. In the hippocampal formation, i.c.v. colchicine injections cause apoptotic death of DGCs, peaking at $40-48 \mathrm{~h}$ (Goldschmidt and Steward 1980; Ceccatelli et al. 1997; Liu et al. 2001). The mode of death is classical apoptosis, including activation of caspase-3 (Bonfoco et al. 1995; Leist et al. 1997), compaction and fragmentation of chromatin (Liu et al. 2001), and display of conspicuous oligonucleosomal bands in gel electrophoresis (Bonfoco et al. 1995; Liu et al. 2001). Up-regulation of c-Jun (Pozas et al. 1999) and cycloxygenase-2 (Ho et al. 1998) at both the mRNA and protein levels precede colchicine-induced DGC apoptosis. However, little knowledge exists regarding the upstream signal transduction pathways leading to activation of programmed cell death following colchicine toxicity.

The c-jun N-terminal kinase (JNK) pathway consists of a cascade of protein kinases that mediates stress signals via activation of mitogen-activated protein kinase kinase (MKK) 4 and MKK7, the upstream kinases of JNK. Activation of the latter finally leads to phosphorylation of the transcription factor c-Jun (reviewed in Ham et al. 2000 and Wang et al. 2004). JNK pathway activation is central to apoptotic neuronal death in several in vitro and in vivo models (Harper and LoGrasso 2001). Furthermore, activation of the JNK pathway has been demonstrated in an animal model of $\mathrm{AD}$ (Savage et al. 2002) and it is essential to beta-amyloidinduced apoptosis in vitro (Troy et al. 2001). In human postmortem brain sections from AD patients, p-JNK has been detected in vulnerable neuronal populations (Atzori et al. 2001; Pei et al. 2001; Zhu et al. 2001). The most prominent substrate of JNK phosphorylation is the Nterminal transactivation domain of c-Jun (Herdegen et al. 1997; Ham et al. 2000). Phosphorylated c-Jun is able to translocate to the nucleus, where it facilitates the transcription of several pro-apoptotic genes of the BH3-only Bcl-2 family, such as Bim and DP5 (Harris and Johnson 2001; Whitfield et al. 2001), Fas (Kuan et al. 2003), and Fasligand (Le-Niculescu et al. 1999). Phosphorylation and translocation of c-Jun is a prerequisite for neuronal apoptosis in several models of apoptotic neuronal death (Estus et al. 1994; Ham et al. 1995; Behrens et al. 1999). Moreover cJun phosphorylation occurs in vulnerable cell populations in animal models of neuronal death (Matsuoka et al. 1999; Vaudano et al. 2001).

CEP-1347 is a mixed-lineage-kinase (MLK) inhibitor that blocks activation of the JNK pathway and protects neurons against apoptotic insults in a number of in vitro and in vivo models (Glicksman et al. 1998; Maroney et al. 1999; Saporito et al. 1999; Bozyczko-Coyne et al. 2001; Boll et al. 2004).
Notably, this inhibitor does not inhibit JNK activity directly, but rather acts upstream by inhibiting the family of MLKs, a class of mitogen activated protein kinase kinase kinases (MAPKKK) that activate MKK4/7 as consequence of stress and cytokine signaling (Maroney et al. 2001).

A critical role for MLK activation in JNK-mediated neuronal death has previously been demonstrated ( $\mathrm{Xu}$ et al. 2001). However, the relative importance of the different MAPKKKs in activating JNK in vivo is still uncertain. Here, we describe activation of JNK signalling after colchicine insult both in vitro and in vivo. We examined whether inhibition of upstream MLKs prevents JNK pathway activation and concomitant apoptosis in granule cells after cytoskeletal disruption by colchicine.

\section{Experimental procedures}

\section{Animals}

Seven-day-old specific pathogen-free BALB/c mice were obtained from Harlan (Horst, the Netherlands). Adult male Wistar rats, weighing 250-300 g, were obtained from Charles River (Sulzfeld, Germany). All animal experiments were performed in accordance with the guidelines of the Danish National Committee on Animal Research Ethics and the European Communities Council Directive \#86/609 for the Care of Laboratory Animals.

\section{Cell culture experiments}

Murine CGCs were isolated and cultured as described previously (Volbracht et al. 1999). Briefly, the neurons were plated in poly-Llysine $(50 \mathrm{mg} / \mathrm{L})$ coated 6/24-well plates at a density of 1800000 cells $/ \mathrm{mL}$ and cultured in basal medium (Eagle's) (BME) (Biowest, Nuaillé, France), supplemented with $10 \%$ heat inactivated fetal calf serum, $2 \mathrm{~mm}$ L-glutamine, $20 \mathrm{~mm} \mathrm{KCl}$ and $1 \%$ penicillin-streptomycin (Gibco-BRL Invitrogen, San Diego, CA, USA). After $24 \mathrm{~h}$, $10 \mu \mathrm{M}$ cytosine arabinoside was added. At 5 days in vitro (DIV), the medium was replaced with a controlled salt solution $(120 \mathrm{~mm} \mathrm{NaCl}$, $25 \mathrm{~mm}$ HEPES, $25 \mathrm{~mm} \mathrm{KCl}, 1.8 \mathrm{~mm} \mathrm{CaCl} 2,4 \mathrm{~mm} \mathrm{MgCl}_{2}, 15 \mathrm{~mm}$ glucose) and cultures were exposed to $1 \mu \mathrm{M}$ colchicine (C-9754, Sigma-Aldrich, St. Louis, MO, USA) and different concentrations of CEP-1347.

For Western blotting, the cells were lysed after 8 or $24 \mathrm{~h}$ colchicine exposure in $200 \mu \mathrm{L}$ lysis buffer containing $10 \mathrm{~mm}$ Tris$\mathrm{HCl}$ (pH 7.2), 1\% NP40, 1 mm 4-(2-Aminoethyl) benzene sulfonyl fluoride hydrochloride (AEBSF), complete protease inhibitor cocktail (cat. no. 1697 498, Roche, Indianapolis, IN, USA) and $6 \mu \mathrm{g}$ protein was then separated on $10 \%$ sodium dodecyl sulfatepolyacrylamide gels. Proteins were transferred to polyvinylidene difluoride membranes and probed with antibodies against p-c-Jun (1:1000; cat. no. SC-822 Santa Cruz Biotechnology, Santa Cruz, CA, USA), c-jun (cat. no. SC-45, Santa Cruz Biotechnology) and fodrin (1:1000; cat. no. MAB1622, Chemicon, Temecula, CA, USA) followed by a horseradish peroxidase conjugated anti-mouse or anti-rabbit antibody (DakoCytomatation, Glostrup, Denmark). Labeled proteins were detected using ECL-Plus reagent (Amersham Life Sciences, Arlington Heights, IL, USA) and developed on film (HyperMax film, Amersham Life Sciences). 
For immunocytochemistry (ICC), cells were fixed in $3.7 \%$ formaldehyde and subsequently labelled with an anti-p-c-Jun antibody according to the manufacturer's protocol ('c-Jun activation hit kit', Cellomics, Pittsburgh, PA, USA). Immunoreactivity was visualized using an Alexa 488-conjugated secondary antibody, which was included in the kit. The nuclei were stained with the fluorescent dye Hoechst-33342, which was also provided in the kit. Nuclear and p-c-Jun fluorescence was digitally imaged with a CCD camera based scanner. The p-c-Jun intensity of nuclei was determined by an image-processing algorithm using the 'Target Activation' software ArrayScan HCS system (Cellomics). Briefly, individual cells were automatically identified by the software and the nuclear area was identified by the pixels positive for H-33342. Then the p-c-Jun immunofluorescence of the nuclear area was integrated for $200-800$ cells per well and the data from eight wells were averaged for one data point.

To quantify the effect of CEP-1347 on colchicine-induced cell death, the viability was measured using the 3-(4,5-demethylthiazole2-yl)-2,5-diphenyltetrasodium bromide (MTT) reduction assay. Furthermore, cells were stained with H-33342 (2 mg/mL) (Molecular Probes, Leiden, Netherlands) as described before (Volbracht et al. 2001) and the number of apoptotic nuclei was scored by use of fluorescence microscopy. Cells with condensed or fragmented nuclei were recognized as apoptotic.

\section{Intracerebroventricular colchicine administration}

Rats were anaesthetized with a mixture of Hypnorm (fentanyl/ fluanisone, Janssen Pharmaceutica, Beerse, Belgium) and Dormicum (midazolam, Hoffmann-La Roche, Basel, Switzerland). The anaesthetic mixture was prepared with 25\% Hypnorm and 25\% Dormicum in sterile distilled $\mathrm{H}_{2} \mathrm{O}$ and administered $2.7 \mathrm{~mL} / \mathrm{kg}$ body weight subcutaneously (s.c.). The anaesthetized rats were then placed in a stereotaxic apparatus with the tooth bar at $-3.3 \mathrm{~mm}$ (Kopf, Tujunga, CA, USA). A scalp incision was made along the midline and a burr hole was drilled in the skull at the coordinates: $-0.9 \mathrm{~mm}$ anterposterior; $1.5 \mathrm{~mm}$ mediolateral (relative to bregma). The dura mater was punctured and a 31-gauge canula lowered to $3.5 \mathrm{~mm}$ dorsoventral from pia mater. Ten microliters of colchicine (dissolved in sterile saline) were injected into the right lateral ventricle through the canula, using a microsyringe (Hamilton, Reno, NV, USA) connected to a microinfusion pump (CMA 100, Carnegie Medicine, Sweden) over a period of $12 \mathrm{~min}$. Sham-operated rats received $10 \mu \mathrm{L}$ sterile saline instead. After injection, to prevent overflow, the canula was left in place for $5 \mathrm{~min}$ and then slowly retracted.

Following infusion, the scalp was sutured, the animal was given analgesic treatment $(0.3 \mathrm{~mL} / \mathrm{kg}$ s.c. Temgesic, buprenorphine, Schering-Plough, Brussels, Belgium), and kept under a warming lamp over night. No general symptoms were detected. In animals used for immunohistochemistry (IHC), injection sites were inspected during processing and animals without a clear needle trace to the lateral ventricles were excluded from the experiment. For the pilot study, the animals were injected with $20 \mu \mathrm{g}$ colchicine. For the dose-response study, the animals received 5,10 or $20 \mu \mathrm{g}$ colchicine to determine the effects of colchicine on activation of JNK pathway. In subsequent experiments animals received $10 \mu \mathrm{g}$ colchicine, as this dose was found sufficient to induce activation of the JNK pathway. Previous studies (unpublished data, G. J. Muller) have shown that $10 \mu \mathrm{g}$ colchicine injected i.c.v. produces about $30 \%$ loss of DGC after $48 \mathrm{~h}$; similar findings were reported by others (Liu et al. 2001).

\section{In vivo inhibition of mixed lineage kinase activation after colchicine injections}

CEP-1347 was kindly provided by Cephalon (West Chester, PA, USA) and prepared as a stock of $6 \mathrm{mg} / \mathrm{mL}$ in $30 \%$ solutol/ phosphate-buffered saline. On the day of use it was diluted to the working concentration of 0.3 or $1 \mathrm{mg} / \mathrm{mL}$ in $5 \%$ solutol/phosphatebuffered saline.

For the in vivo kinase inhibition experiment, CEP-1347 solution was administered s.c. at $1 \mathrm{~mL} / \mathrm{kg}$. Rats received one dose of CEP$13471 \mathrm{~h}$ prior to colchicine injection, a second dose $6 \mathrm{~h}$ after the colchicine injection and a third dose $24 \mathrm{~h}$ after the first dose, $2 \mathrm{~h}$ before termination. For the in vivo neuroprotection study, where the rats survived for $48 \mathrm{~h}$ after colchicine injection, the rats were treated as in the kinase inhibition experiment during the first $24 \mathrm{~h}$ and then twice daily. Vehicle-treated rats received $1 \mathrm{~mL} / \mathrm{kg} 5 \%$ solutol/ phosphate-buffered saline at the same time points.

\section{Tissue processing}

Rats were deeply anaesthetized with an overdose of Avertin (Bie \& Berntsen, Roedovre, Denmark, $10 \mathrm{~mL} / \mathrm{kg}$ ). For western blotting, the animals were decapitated, brains were extracted from the skull and the anterior part of the dentate gyrus (DG) was dissected and frozen immediately on pulverized dry ice, then stored at $-20^{\circ} \mathrm{C}$. For histology, the anaesthetized animals were perfusion-fixed transcardially through the ascending aorta with a fixative containing $4 \%$ paraformaldehyde in $0.15 \mathrm{M}$ phosphate buffer $\left(\mathrm{pH} 7.3\right.$ at $\left.4^{\circ} \mathrm{C}\right)$. Brains were postfixed in the same fixative for $4 \mathrm{~h}$ at room temperature, cryoprotected in $30 \%$ sucrose in $0.12 \mathrm{~m}$ phosphate buffer before they were frozen on dry ice. Parallel series, each consisting of six $30-\mu \mathrm{m}$ thick coronal sections, were cut on a cryostat at coronal brain level $-2.0 \mathrm{~mm}$ to $-4.0 \mathrm{~mm}$ relative to bregma. The sections were then processed for hematoxylin/eosin $(\mathrm{H} / \mathrm{E})$ staining or IHC against p-c-Jun.

\section{Western blotting procedure}

Tissue samples were sonicated in a buffer consisting of $20 \mathrm{~mm}$ HEPES pH 7.4, 2 mм EGTA, 50 mm $\beta$-glycerolphosphate, $10 \%$ glycerol, $1 \mathrm{~mm} \mathrm{Na} \mathrm{VO}_{4}, 50 \mathrm{~mm} \mathrm{NaF}$, complete protease inhibitor cocktail (Roche), AEBSF $1 \mathrm{~mm}$ (Calbiochem), 1\% Triton-X100. The samples were incubated on ice for $15 \mathrm{~min}$, centrifuged at $1000 \mathrm{~g}$ for $10 \mathrm{~min}$ and the supernatant collected for further analysis. Protein concentration in the homogenate was determined with a BCA protein assay kit (Pierce, Rockford, IL, USA). Thirty micrograms of protein from each sample were run in duplicates on NuPAGE Bis-Tris 10\% acrylamide gels under reducing conditions, according to the manufacturer's instructions (Invitrogen, San Diego, CA, USA). Following gel electrophoresis, proteins were transferred to a polyvinylidene difluoride membrane using the Xcell SureLock Mini-Cell system (Invitrogen). For immunological detection of protein bands, the membranes were probed with primary antibodies against p-c-jun (1:1000, Santa Cruz Biotechnology) in Tris-buffered saline with Tween containing 3\% skimmed milk powder over-night at $4^{\circ} \mathrm{C}$. After washing, the membranes were incubated with horseradish peroxidase conjugated anti-mouse 
secondary antibody at a dilution of 1:5000 (cat. no. P0260, DakoCytomatation). The signal was detected using Super Signal West Dura extended duration substrate (Pierce) and captured with a digital camera using the LAS-3000 Fujifilm ImageReader system.

\section{Western blot analysis}

Images were imported into the AlphaEase analysis software (AlphaInnotec, San Leandro, CA, USA). The optical densities of bands representing the proteins of interest were then measured, normalized to protein amount and the values for each of the duplicate determinations were averaged to provide a measure of phosphorylation of c-jun in each sample. All samples were quantified against a four-fold dilution series of rat hippocampal homogenate run on each gel. This dilution series serves as an internal standard curve on each gel and can be used to ensure that the signals measured are within the linear range of detection for each protein of interest. The vehicle effect on phosphorylation of c-jun was subtracted in samples from the same experimental day and differences between experimental groups were analyzed by one-way ANOva and Tukey test.

\section{Immunohistochemistry}

IHC was performed free-floating on serial sections, applying a polyclonal antibody against phospho-c-Jun Ser63 (p-c-Jun, cat. no. 9261, Cell Signaling Technology, Beverly, MA, USA); a polyclonal antibody against amino acids 209-225 of c-Jun [c-Jun/AP-1 (Ab-1), cat. no. PC $06 \mathrm{~L}$, Oncogene, Cambridge, MA, USA] and a polyclonal antibody recognizing the site specific Asp 175 cleaved large fragment $(17 / 19 \mathrm{kDa})$ of activated human caspase-3 (cat. no. 9661, Cell Signaling Technology). Briefly, the sections were rinsed $3 \times 5 \mathrm{~min}$ in $0.05 \mathrm{~m}$ phosphate-buffered saline $\mathrm{pH}$ 7.4. Endogenous peroxidase was blocked with $0.3 \%$ hydrogen peroxide $\left(\mathrm{H}_{2} \mathrm{O}_{2}\right)$ in methanol for $20 \mathrm{~min}$. The primary antibody was then applied at concentration (1:100 for p-c-jun and c-jun and 1:300 for activated caspase-3) in phosphate-buffered saline for $24 \mathrm{~h}$ at $4^{\circ} \mathrm{C}$. A secondary biotinylated antibody (1:200) was applied for $1 \mathrm{~h}$ at room temperature. This was followed by incubation with streptavidin-horseradish peroxidase conjugated antibody (1:200, Streptavidin-HRP, DAKO, Denmark, 9-0397) for $1 \mathrm{~h}$ at room temperature. Between each step, sections were rinsed $3 \times 5$ min with $0.05 \mathrm{M}$ phosphate-buffered saline. Sections were then preincubated in DAB (0.05\% 3,3'-diaminobenzidine, Sigma, D-8001) without $\mathrm{H}_{2} \mathrm{O}_{2}$ for $10 \mathrm{~min}$, before $0.015 \% \mathrm{H}_{2} \mathrm{O}_{2}$ was finally added. The peroxidase reaction was stopped after 15 min by adding distilled $\mathrm{H}_{2} \mathrm{O}$. Sections were mounted on gelatin-coated microscopic slides and allowed to dry overnight at $4{ }^{\circ} \mathrm{C}$. They were then counterstained in $1 \%$ cresylviolet for $20 \mathrm{~min}$, dehydrated and passed through xylene from where they were cover slipped with Eukitt. Specific immunostaining was detected as a diffuse brown precipitate. Negative controls, where the primary antibody was omitted, were completely blank.

\section{Stereological methods}

The unbiased optical fractionator method (West and Gundersen 1990; West et al. 1991) was used for sampling and estimation of numbers of positive profiles per unit area ipsilateral to the side of colchicine injection. In our study, every 6th section was sampled and used for stereology. The sections were analysed using the Olympus CAST-system. The border of each DGC layer was outlined using a
$10 \times$ objective. For counting p-c-Jun-positive DGCs, apoptotic profiles or intact DGCs, a $100 \times$ oil-immersion objective was used. The program created a serie of uniform random systematic sample fields of vision throughout the marked region. Using an unbiased counting frame of $1700 \mu \mathrm{m}^{2}$ and $x$ and $y$-step lengths of $100 \mu \mathrm{m}$, we created an average of 26 systematic sampling fields of vision (counting frames) throughout the DGC layer on one section which resulted in a minimum of 200 counted objects (cells) per DGC layer and section. For estimation of positive profiles number/unit area, the total number of objects in all dissectors counted on each section was divided by the total volume of these dissectors. Average cell number/unit area was then generated from the five most anterior sections, as the anterior DG is particularly sensitive to colchicine toxicity (Liu et al. 2001). The average of each group was calculated and the results were then analysed by ANOvA followed by NewmanKeuls test. Statistical significance was accepted for $p<0.05$.

\section{Results}

\section{C-Jun phosphorylation and nuclear translocation in cultured cerebellar granule cells after colchicine treatment}

Incubation of murine CGCs with $1 \mu \mathrm{m}$ colchicine led to activation of the JNK pathway within $8 \mathrm{~h}$ (the earliest time point that the cytoskeletal changes is observable) as shown by phosphorylation of c-Jun with no concomitant change in unphosphorylated c-jun levels (Fig. 1a). C-Jun is sitespecifically (Ser63, Ser73) phosphorylated by p-JNK (Kallunki et al. 1994; Minden et al. 1994) and thus can be used as a footprint for activation of the entire JNK pathway. The data obtained by Western blotting were confirmed by quantitative single cell immunofluorescence analysis, which showed a clear increase in p-c-Jun translocation from cytoplasm to the nucleus at the same time point (Fig. 1b). To correlate c-Jun activation with another intracellular event, triggered by colchicine, we measured the proteolysis of fodrin. Both $\mu$-calpain and activated caspase- 3 cleave native fodrin $(240 \mathrm{kDa})$ into a common $(150 \mathrm{kDa})$ fragment. Moreover, caspase-3 activation leads to a specific cleavage product of $120 \mathrm{kDa}$ size (Nath et al. 1996; Volbracht et al. 1999). As expected, we observed an increase in both types of cleavage fragments after colchicine treatment (Fig. 1a).

\section{Effects of mixed lineage kinase inhibition on activation of the c-Jun N-terminal kinase pathway and apoptosis induced by colchicine in cerebellar granule cell cultures We investigated whether upstream activation of MLKs was involved in the phosphorylation of c-Jun and in fodrin proteolysis triggered by colchicine. As a tool we used the potent MLK inhibitor CEP-1347. We found, both by Western blotting and ICC, that CEP-1347 at concentrations as low as $100 \mathrm{~nm}$ significantly inhibited the activation of c-Jun (Figs 1a and b). Furthermore, inhibition of colchicine-}



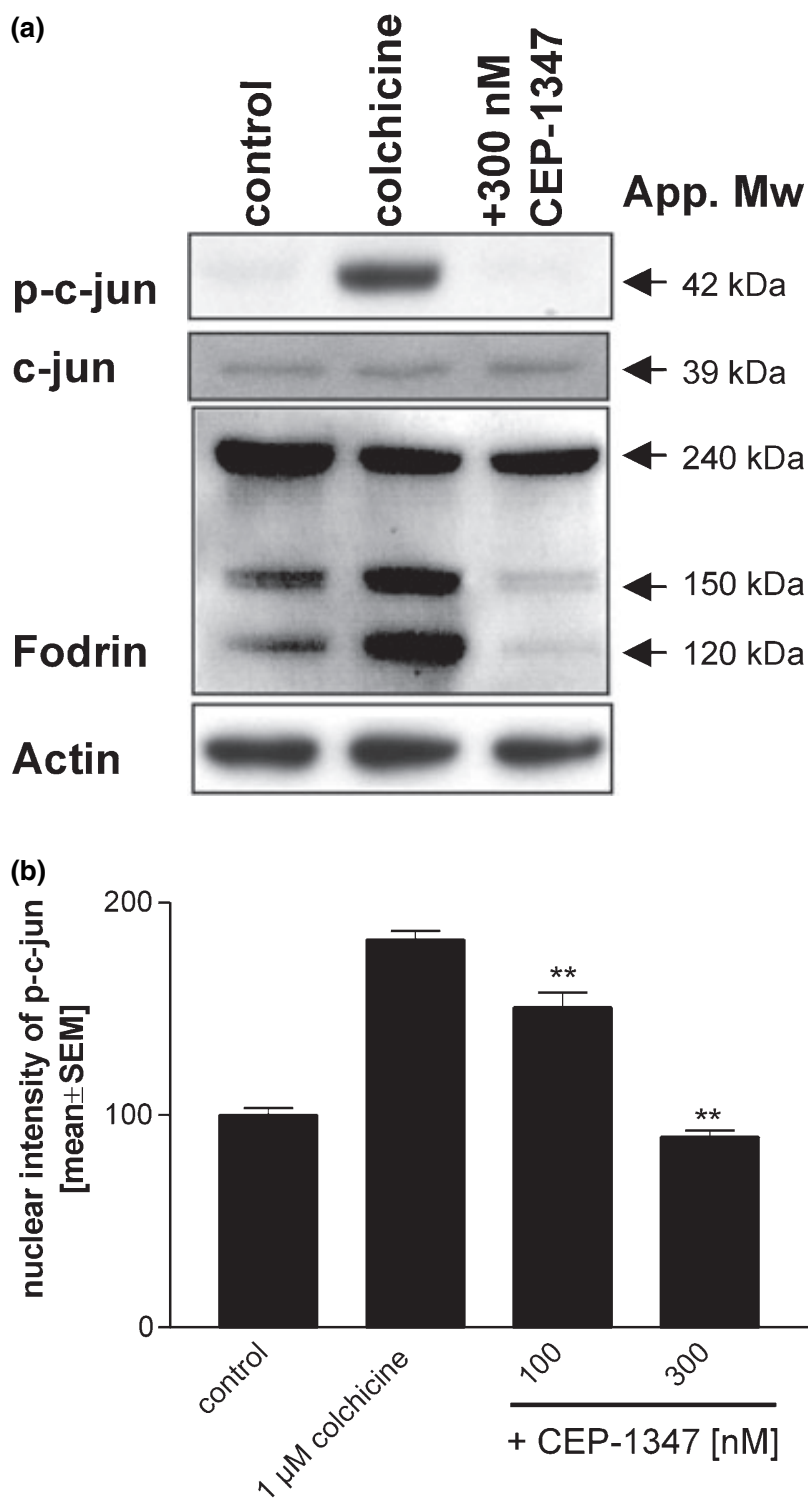

Fig. 1 Effect of CEP-1347 on activation of c-jun and caspase3-mediated fodrin-cleavage. (a) Cerebellar granule cells (CGCs) were incubated with CEP-1347 or vehicle and challenged with $1 \mu \mathrm{m}$ colchicine before being lysed and analysed after $8 \mathrm{~h}$ for $\mathrm{p}$-c-Jun and c-jun or after $24 \mathrm{~h}$ for fodrin by western blotting. Arrows indicate apparent molecular weight of c-jun (39 kDa), p-c-jun (42 kDa), uncleaved fodrin $(240 \mathrm{kDa})$, the calpain/caspase-3 common $(150 \mathrm{kDa})$ and the caspase-3-specific (120 kDa) cleavage products. (b) Colchicine-treated CGCs were fixed after $8 \mathrm{~h}$ and immunostained for $\mathrm{p}$-c-Jun. The nuclear staining intensity of $\mathrm{p}$-c-Jun was determined by automatic digital imaging analysis of the fluorescent staining in the nucleus. Data represent quantifications from $n=8$ wells with 200-800 cells analyzed per well and normalized to control (mean \pm SEM). CEP-1347 significantly decreased the nuclear staining intensity $\left({ }^{* *} p<0.01\right.$; oneway ANOva followed by the Tukey test).

induced c-Jun activation by CEP-1347 was paralleled by prevention of caspase-3/calpain-mediated cleavage of fodrin (Fig. 1a). We further investigated whether the prevention of the JNK activation by CEP-1347 also resulted in cytoprotection. Indeed, CEP-1347 protected CGCs from colchicineinduced cell death quantified either by a tetrazolium reduction assay (Fig. 2a) or by counting of apoptotic nuclei (Fig. 2b). The concentrations effective at reducing apoptosis (100-300 nM) were similar to those blocking the JNK pathway activation. Colchicine-treated cells display a caspase-independent marked loss of neurite extensions that precedes apoptosis (Fig. 1c) (Volbracht et al. 1999, 2001). Cultures treated with $300 \mathrm{~nm}$ CEP-1347 showed a reduced disintegration of neurites (Fig. 1c), suggesting a role of JNK also in caspase-independent death routines.

\section{Pilot study to determine if intracerebroventricular} colchicine administration leads to activation of the c-Jun $\mathrm{N}$-terminal kinase pathway in vivo

In a first pilot study, adult rats received a dose $(20 \mu \mathrm{g})$ of colchicine that was chosen from the literature (Ceccatelli et al. 1997). In addition, one group of animals was cotreated with colchicine and CEP-1347 (1 mg/kg). At $24 \mathrm{~h}$ after colchicine injection, the brains were prepared for IHC and examined for the presence of markers of activation of the JNK pathway and of apoptosis in hippocampal dentate gyrus granule cells. Total c-Jun was clearly visible in nuclei of sham-operated animals (Fig. 3a), and was strongly induced in animals that received colchicine (Figs $3 b$ and $c$ ). The phosphorylated form, p-c-Jun, was not detected in shamoperated animals (Fig. 3d), but was induced in nuclei of animals that received colchicine (Figs $3 \mathrm{e}$ and $\mathrm{f}$ ). Treatment with CEP-1347 (1 mg/kg) showed a trend towards decreasing the number of $\mathrm{p}$-c-jun-positive neurons, although this was not significant (Fig. 3m). Activated caspase-3 was detected in dendrites, cytoplasm and nuclei of granule cells in colchicinetreated animals (Figs $3 \mathrm{~h}$ and i), but not in sham-operated animals (Fig. 3g); caspase-3 immunoreactivity was detected in both intact neurons and in neurons with strongly condensed cytoplasm and chromatin. H/E staining revealed the beginning of cellular shrinkage, associated with eosinophilia, and the formation of nuclear chromatin clumps, with increased basophilia in rats injected with colchicine (Fig. 3k). Treatment with CEP-1347 had no obvious effect on the cellular changes in H/E stained sections (Fig. 31). Thus, colchicine at a dose of $20 \mu \mathrm{g}$ induced activation of the JNK pathway in hippocampal granule cells and triggered apoptosis. Individual animals treated with colchicine were followed over longer time periods, and $\mathrm{H} / \mathrm{E}$ staining in these animals showed that apoptosis was more pronounced at $48 \mathrm{~h}$ (data not shown).

\section{In vivo dose-response of c-Jun $\mathrm{N}$-terminal kinase} activation in the dentate gyrus after colchicine intracerebroventricular injection

To optimize the experimental protocol, different doses of colchicine were tested for their capacity to induce the JNK pathway. For this purpose, rats were injected with 5,10 or 

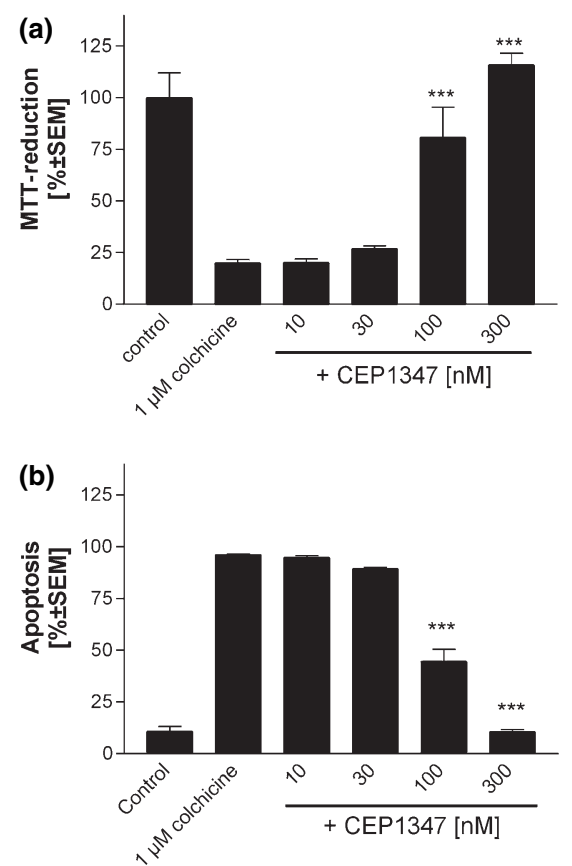

(c)

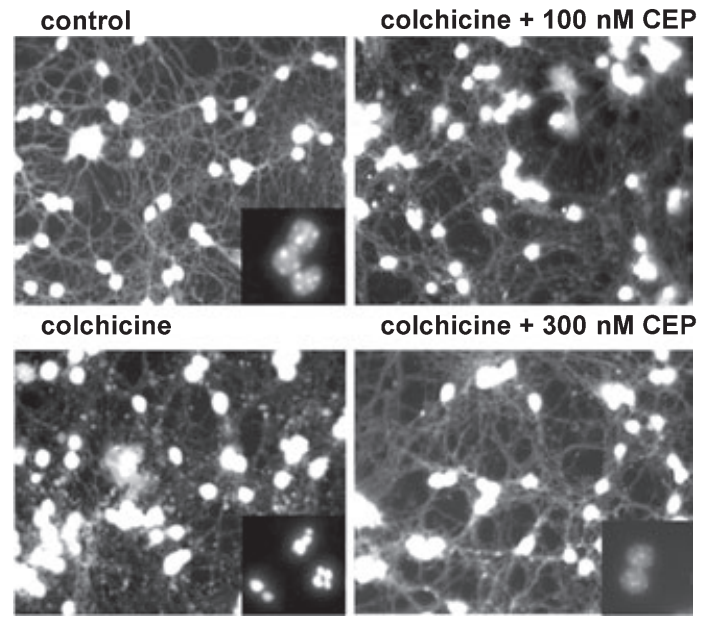

Fig. 2 Effect of CEP-1347 on colchicine-induced granule cell death in vitro. Cerebellar granule cells (CGCs) were incubated with CEP1347 or vehicle and challenged with $1 \mu \mathrm{m}$ colchicine for $24 \mathrm{~h}$ (a) Cell viability was measured using the MTT reduction assay. (b) In parallel cultures cells were stained with the fluorescent dye Hoechst-33342 and the number of apoptotic nuclei was scored by use of fluorescence microscopy. Cells with condensed and fragmented nuclei were scored as apoptotic. Data are presented as percentage of total number of cells (mean \pm SEM). (c) Morphology of cultured CGCs in the presence of colchicine and CEP-1347. CGCs treated with vehicle (upper left picture), $1 \mu \mathrm{m}$ colchicine (lower left picture) or colchicine plus $100 \mathrm{~nm}$ (upper right picture) or $300 \mathrm{~nm}$ (lower right picture) CEP-1347 for $24 \mathrm{~h}$. The cultures were stained with Calcein-AM for visualization of effects on the neuritic network or Hoechst-33342 (inserts) to show the nuclear morphology. CEP-1347 significantly increased viability and decreased the percentage of apoptotic nuclei $\left(^{\star * *} p<0.001\right.$; one-way ANOVA followed by the Tukey test, $n=3$ ). CEP-1347 did not change basal MTT turn over.
$20 \mu \mathrm{g}$ colchicine or saline and killed $25 \mathrm{~h}$ later. Western blotting for p-c-Jun was used as a read out for the activation of the JNK pathway and a dose of $10 \mu \mathrm{g}$ colchicine was found to be optimal. Under this condition p-c-Jun was increased eightfold in homogenates from the hippocampus (Fig. 4). For the following experiments, we chose to continue with this dose, since it produced a robust signal and no side-effects in the animals, even over several day-long observation periods.

\section{Effects of mixed lineage kinase inhibition on in vivo colchicine-induced JNK pathway activation}

We investigated the effect of inhibition of the MLKs by CEP1347 using the optimized colchicine-dosing schedule. Groups of rats received $10 \mu \mathrm{g}$ colchicine i.c.v. and were treated with 0.3 or $1 \mathrm{mg} / \mathrm{kg}$ CEP-1347 to evaluate a dosedependency of the pharmacological treatment. After $25 \mathrm{~h}$, brains were prepared for analysis by immunoblotting, and CEP-1347 (0.3 or $1 \mathrm{mg} / \mathrm{kg}$ ) reduced phoshorylation of c-Jun significantly in a dose-dependent manner (Fig. 5).

\section{Effects of mixed lineage kinase inhibition on neuronal death induced in vivo by colchicine administration} In a last set of experiments, we examined whether MLKinhibition by CEP-1347 would lead to neuroprotection in the in vivo colchicine i.c.v. injection model. Rats received $10 \mu \mathrm{g}$ colchicine and cell death was evaluated at the optimal time point of $48 \mathrm{~h}$ after the challenge. In this experiment, two dose groups of CEP-1347 were included $(0.3$ or $1 \mathrm{mg} / \mathrm{kg}$ ) to study potential dose-dependent effects.

IHC analysis showed that colchicine strongly increased p-c-Jun in intact appearing nuclei of DGCs, whereas no reactivity was detected in DGCs of sham-operated animals (Fig. 6a). Furthermore, we observed a dose-dependent decrease in the number of p-c-Jun immunopositive neurons in CEP-1347-treated animals compared to vehicle-treated ones. However, the decrease was not significant, which may be due to the relatively small experimental groups $(n=6)$ and the more complex pathological situation at the $48 \mathrm{~h}$ time point.

In the same sections, examined by using an unbiased stereological method, we found that colchicine treatment caused a strong, significant increase in the number of apoptotic DGCs (Fig. 6b), and a significant decrease in the number of intact DGCs (Fig. 6c) per unit area. Treatment with CEP-1347 (0.3 and $1 \mathrm{mg} / \mathrm{kg})$ dose-dependently reduced the number of apoptotic profiles and the effect of $1 \mathrm{mg} / \mathrm{kg}$ drug was significant when compared to animals receiving vehicle $(p<0.05$, Fig. 6b). This protective effect was corroborated by the finding of more surviving cells in the CEP-1347 groups ( $p<0.01$ at $1 \mathrm{mg} / \mathrm{kg}$, Fig. $6 \mathrm{c}$ ).

\section{Discussion}

In the present study, we demonstrate that the JNK pathway is activated in colchicine-mediated apoptotic neuronal death 

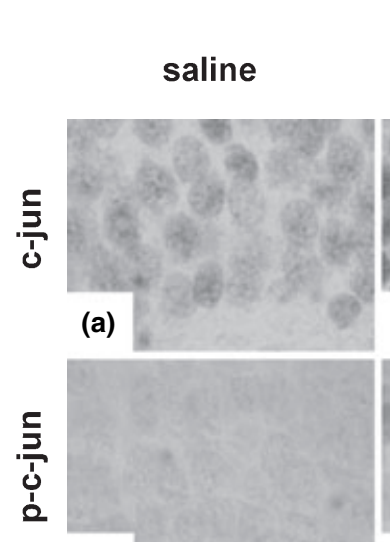

(d)
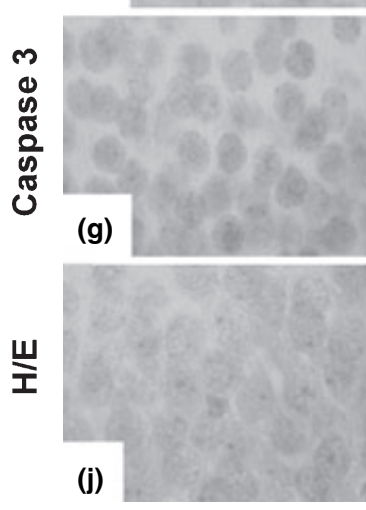

(h)
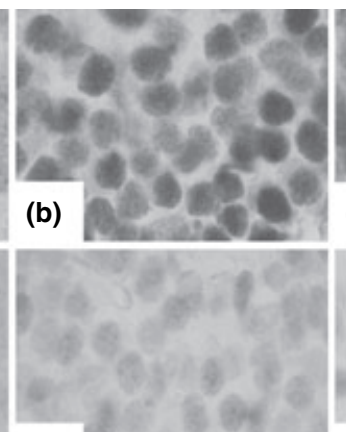

(e)
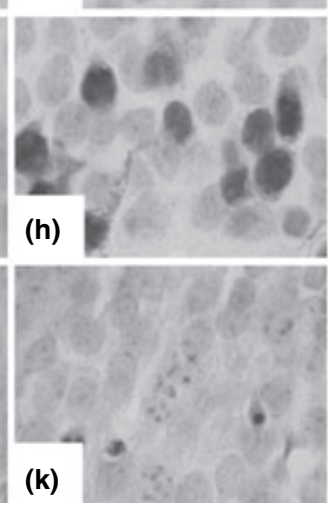

(i)
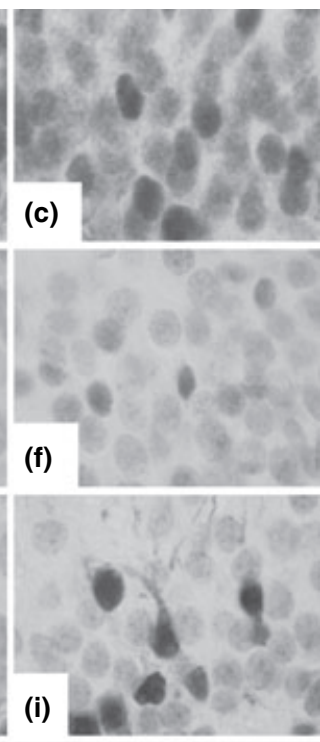

(m)

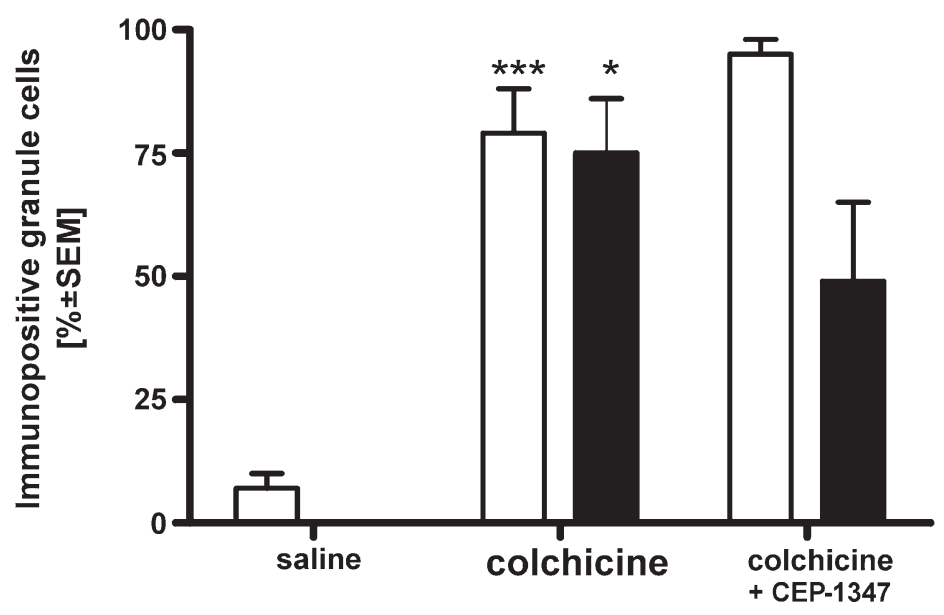

Fig. 3 Staining of the dentate gyrus for c-Jun, p-c-Jun, caspase-3 and hematoxylin/ eosin $(H / E) 24 \mathrm{~h}$ after colchicine injection. In this pilot experiment, rats $(n=4$ per group) were injected with $20 \mu \mathrm{g}$ colchicine and treated with vehicle or $1 \mathrm{mg} / \mathrm{kg}$ CEP1347. After $24 \mathrm{~h}$, the animals were perfusion fixed and the brains were cut into sections, processed for c-Jun, p-c-Jun or activated caspase-3 immunohistochemistry or $H / E$ staining. C-Jun antibodies labelled granule cell nuclei in sham-operated rats (a) and was strongly induced in animals receiving colchicine (b, c). p-c-Jun (Ser63) immunoreactivity was absent in controls (d) and localized in nuclei after colchicine injections (e, f). Caspase- 3 was absent in controls (g) and strongly labelled dendrites, soma and nuclei of granule cells $(h, i)$. In sections stained with $\mathrm{H} / \mathrm{E}$, beginning cellular shrinkage, associated with eosinophilia, and formation of nuclear chromatin clumps, with increased basophilia, were detected after colchicine injections (k, I). Bar: $50 \mu \mathrm{m}$. Quantification of the number of $c$-jun and p-c-jun-positive neurons (m). Data represent mean relative numbers (\%) of immunopositive dentate granule cells counted in a total area of $0.03 \mathrm{~mm}^{2}$ in the ventral blade of the granule cell layer. c-jun and p-c-Jun were significantly induced by colchicine treatment. $\left({ }^{*} p<0.05 ;{ }^{* * *} p<0.001\right.$ anova followed by Dunnett's method). both in vitro and in vivo. Moreover, we find that inhibition of upstream MLKs by CEP-1347 prevents activation of the JNK pathway and prevents both CGCs and DGCs from undergoing apoptotic cell death.

In agreement with previous studies (Bonfoco et al. 1995; Ceccatelli et al. 1997; Volbracht et al. 1999), both CGCs in vitro and DGCs in vivo underwent classical apoptosis mediated by caspase-3 activation upon colchicine exposure. JNK pathway activation and phoshorylation/nuclear trans- location of c-Jun preceded apoptosis in both CGC and DGC death. In CGCs exposed to colchicine, MLK inhibition by CEP-1347 led to a blockade of c-Jun phosphorylation/nuclear translocation, prevented caspase-3 activation, as evidenced by lack of fodrin cleavage, and protected cells from apoptosis. Thus, MLK-mediated activation of the JNK pathway leading to c-Jun phosphorylation is critical for $\mathrm{CGC}$ apoptosis induced by cytoskeletal damage. 


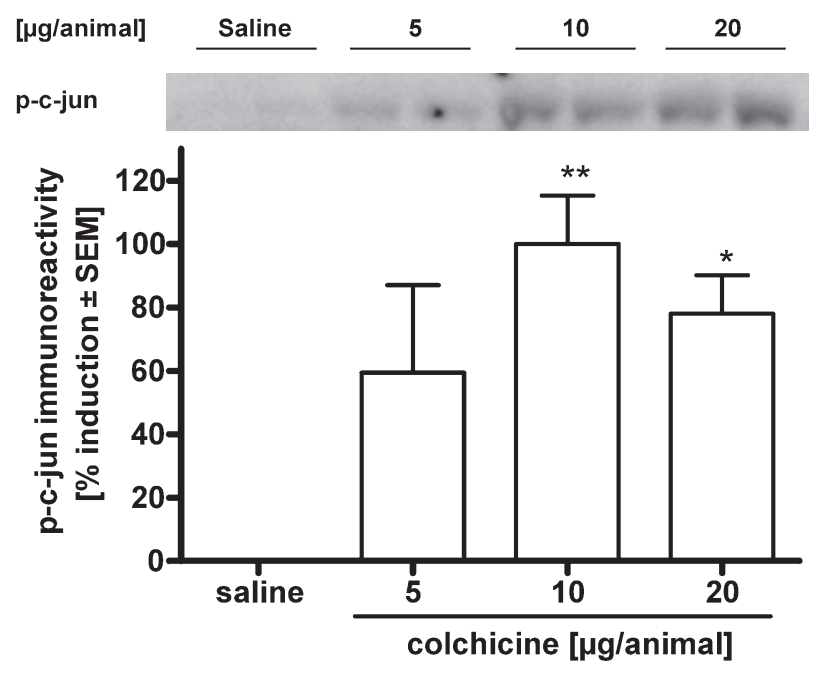

Fig. 4 Immunolabeling of p-c-jun in the dentate gyrus after intracerebroventricular colchicine injections. Top panel: western blot of $p-c-$ jun. Samples are loaded in duplicates. Lower panel: quantification of western blots. Animals were injected with $0,5,10$ or $20 \mu \mathrm{g}$ colchicine and after $25 \mathrm{~h}$ the tissue was dissected and analysed for $\mathrm{p}$-c-jun by western blotting. Lysates from each animal were loaded in duplicates. The duplicate determinations were averaged to provide a measure of phosphorylation of c-jun in each sample, and the average for all animals in each group is presented in the graph. The signals are normalized to protein levels and the levels are standardized to saline as basal level and maximal colchicine induction as $100 \%$. Data represent the mean relative increase (mean \pm SEM) in optical densities from four animals per group $(n=4)$ (one-way ANOvA followed by Tukey test, $\left.{ }^{*} p<0.05 ;{ }^{* *} p<0.01\right)$.

Cultured CGCs show high constitutive levels of JNK activity, with different pools of JNKs serving different functions (Coffey et al. 2000). Stress induced by the general phosphatase inhibitor calyculin A specifically activates JNK2/3, which then is responsible for c-Jun phosphorylation (Coffey et al. 2002) in the presence of a constitutive JNK1 activity. MLK inhibitors act specifically upon the suprabasal stress-induced JNK activity (mainly JNK2/3), leaving constitutive JNK (mainly JNK1) activity untouched (Mielke and Herdegen 2000). If this applies also to the CGC model used in our study, it may explain why in addition to protection from apoptosis, we found that CEP-1347-treated cells maintained mitochondrial function (as shown by the MTT assay) and showed at least a delay in their colchicine-induced neurite fragmentation. Similar effects of CEP-1347 have been described in trophic factor deprived-superior cervical ganglion neurons (Harris et al. 2002), in A $\beta$-induced toxicity in cortical neurons (Bozyczko-Coyne et al. 2001) and in stress models of primary dopaminergic neurons (Boll et al. 2004). Indeed, it has recently been shown that JNK1 (but not JNK2 or 3) activity is required for maintaining cytoskeletal integrity via phosphorylation of microtubule-associated proteins (MAPs) (Chang et al. 2003).

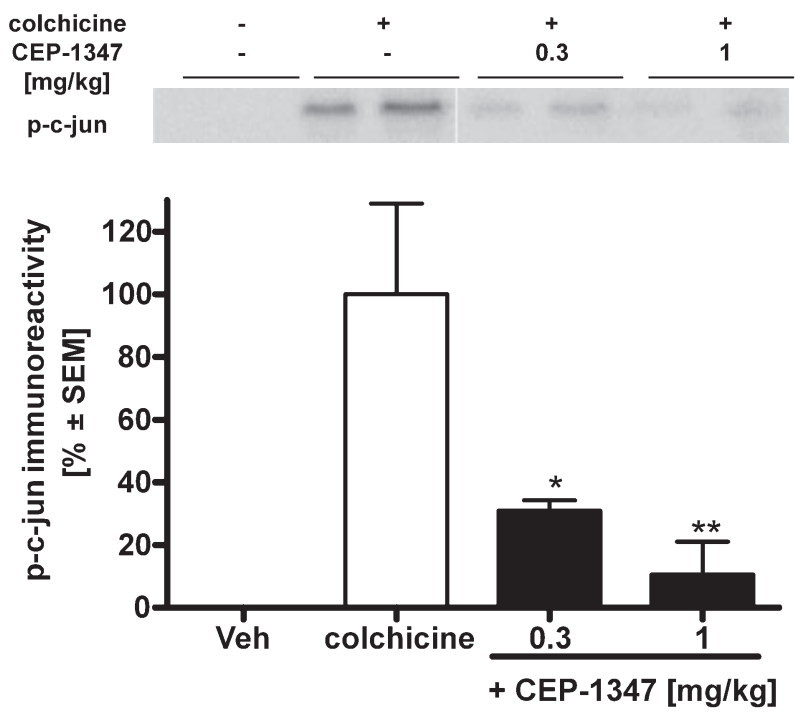

Fig. 5 Effect of CEP-1347 on p-c-jun in the anterior hippocampus after intracerebroventricular injection. Top panel: western blot of $\mathrm{p}$-c-jun. Samples are loaded in duplicates. Lower panel: quantification of western blots. Animals were injected with $10 \mu \mathrm{g}$ colchicine and treated with vehicle, 0.3 or $1 \mathrm{mg} / \mathrm{kg}$ CEP-1347. After $25 \mathrm{~h}$ the tissue was dissected and analysed for $\mathrm{p}-\mathrm{c}$-jun by western blotting. Lysates from each animal were loaded in duplicates. The duplicate determinations were averaged to provide a measure of phosphorylation of c-jun in each sample, and the average for all animals in each group is presented in the graph. The signals are normalization to protein levels and levels are standardized to saline as basal level and maximal colchicine induction as $100 \%$. Data represent the mean relative increase (mean \pm SEM) in optical densities from four animals per group $(n=4)$ (one-way ANOVA followed by Tukey test, ${ }^{*} p<0.05 ;{ }^{* *} p<0.01$ ).

Although there is now clear evidence for the role of MLKs in the control of the JNK pathway in neuronal death models in vitro, limited information is available on the involvement of MLKs in JNK activation in neuronal death models in vivo. In our study MLKs played a role in the activation of the JNK pathway induced by colchicine in DGCs in vivo, leading to c-Jun phosphorylation and apoptosis. Together with an induction of p-c-Jun, total c-Jun was also up-regulated in DGCs in vivo by colchicine, which is in agreement with a previous study (Pozas et al. 1999). Treatment with CEP1347 significantly attenuated phosphorylation of c-Jun seen by western blotting and - although not significantly - the relative number of p-c-Jun-positive DGCs. Furthermore, CEP-1347 significantly decreased the number of apoptotic DGCs and increased the number of intact DGCs. This proves that MLKs indeed are important in mediating colchicineinduced granule cell apoptosis; an effect which, most likely, is mediated through the JNK pathway. A non-significant decrease in the density of p-c-Jun-positive DCG, however, could also be due to the inherent variation of (p-c-Jun) IHC, which would demand large animal groups to be overcome (Vaudano et al. 2001). 

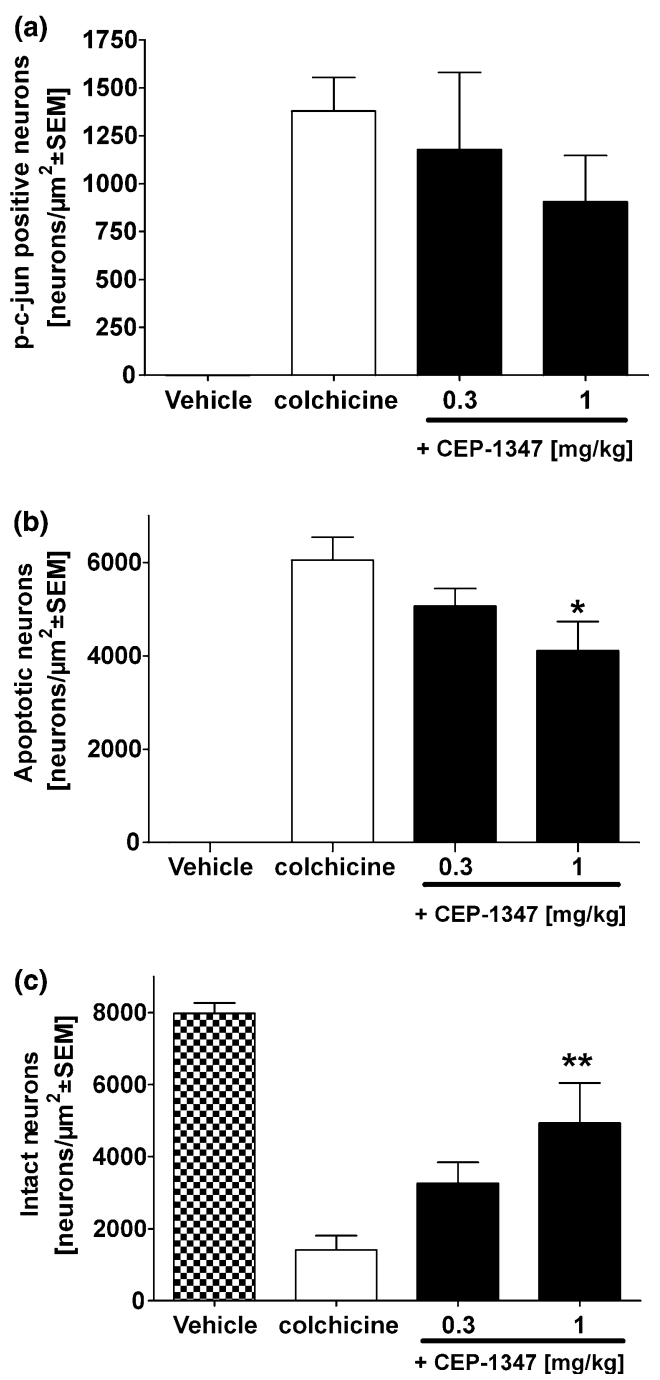

Fig. 6 Effect of CEP-1347 on dentate gyrus granule cell apoptosis induced by colchicine intracerebroventricular injection. Animals were injected with $10 \mu \mathrm{g}$ colchicine and treated with vehicle, 0.3 or $1 \mathrm{mg} / \mathrm{kg}$ CEP-1347. After $48 \mathrm{~h}$ the animals were perfusion fixed and the brains were cut in parallel series, each consisting of six $30-\mu \mathrm{m}$-thick coronal sections. The sections were then processed for immunohistochemistry against $p$-c-Jun or hematoxylin/eosin staining. The number of $p$-c-jun (a), apoptotic dentate granule cells (DGCs) (b) and intact DGCs (c) was counted using the unbiased optical fractionator method. The data are presented as granule cell numbers/unit area for each group (mean \pm SEM). CEP-1347 significantly decreased the number of apoptotic DGCs and increased the number of intact neurons ( ${ }^{*} p<0.05 ;{ }^{* *} p<0.01$; one-way ANOVA followed by Newman-Keuls test).

We have shown that activation of the JNK pathway leading to c-Jun phosphorylation is a critical event in neuronal apoptosis induced by cytoskeletal dysfunction. These results may contribute to our understanding of the molecular events underlying neurodegeneration in human diseases where cytoskeletal dysfunction is a pathogenic factor, such as in $\mathrm{AD}$. Indeed, there is evidence for activation of the JNKs in the brain of AD patients, where it appears to be an event starting early in the disease and following its progression (Pei et al. 2001; Zhu et al. 2001). Interestingly, JNK activation is initially localized to neuronal nuclei of susceptible neuronal populations and later to neurons undergoing neurofibrillary changes and appears to precede the deposition of amyloid in the extracellular space, suggesting that it may be triggered by cytoskeletal alterations (Pei et al. 2001). Also, JNK activation has been detected in mouse models of $\mathrm{AD}$ (Shoji et al. 2000; Savage et al. 2002), where it was localized to dystrophic hyperphosphorylated neurites surrounding amyloid plaques.

We have also found that MLKs are involved in colchicineinduced JNK activation and that MLK inhibition prevents c-Jun phosphorylation and cell death in CGCs and DGCs. In addition, we observed that CEP-1347 preserved neurites after colchicine exposure in CGCs. This is in striking contrast with the effects of caspase inhibitors, which do not protect the neuritic network. This may have important implications for the treatment of neurodegenerative diseases because MLK inhibitors, such as CEP-1347, may not only save dying neurons, but may also maintain their function or even reverse dysfunction. We therefore suggest that prevention of apoptotic signalling by this mechanism may rescue degenerating neurons in diseases such as $\mathrm{AD}$.

\section{Acknowledgements}

We would like to thank Cephalon, Inc. (West Chester, PA, USA) for generously providing CEP-1347 and Anne Klit Thomsen and Karina Petersen (H. Lundbeck, Valby, Denmark) for excellent technical assistance.

\section{References}

Atzori C., Ghetti B., Piva R., Srinivasan A. N., Zolo P., Delisle M. B., Mirra S. S. and Migheli A. (2001) Activation of the JNK/p38 pathway occurs in diseases characterized by tau protein pathology and is related to tau phosphorylation but not to apoptosis. J. Neuropathol. Exp. Neurol. 60, 1190-1197.

Behrens A., Sibilia M. and Wagner E. F. (1999) Amino-terminal phosphorylation of c-Jun regulates stress-induced apoptosis and cellular proliferation. Nat. Genet. 21, 326-329.

Boll J. B., Geist M. A., Kaminski Schierle G. S., Petersen K., Leist M. and Vaudano E. (2004) Improvement of embryonic dopaminergic neurone survival in culture and after grafting into the striatum of hemiparkinsonian rats by CEP-1347. J. Neurochem. 88, 698-707.

Bonfoco E., Ceccatelli S., Manzo L. and Nicotera P. (1995) Colchicine induces apoptosis in cerebellar granule cells. Exp. Cell Res. 218, 189-200.

Bozyczko-Coyne D., O’Kane T. M., Wu Z. L., Dobrzanski P., Murthy S., Vaught J. L. and Scott R. W. (2001) CEP-1347/KT-7515, an inhibitor of SAPK/JNK pathway activation, promotes survival and blocks multiple events associated with Abeta-induced cortical neuron apoptosis. J. Neurochem. 77, 849-863. 
Brück W. and Stadelmann C. (2003) Inflammation and degeneration in multiple sclerosis. Neurol. Sci. 24, 265-267.

Ceccatelli S., Ahlbom E., Diana A. and Zhivotovsky B. (1997) Apoptosis in rat hippocampal dentate gyrus after intraventricular colchicine. Neuroreport 8, 3779-3783.

Chang L., Jones Y., Ellisman M. H., Goldstein L. S. and Karin M. (2003) JNK1 is required for maintenance of neuronal microtubules and controls phosphorylation of microtubule-associated proteins. Dev. Cell 4, 521-533.

Coffey E. T., Hongisto V., Dickens M., Davis R. J. and Courtney M. J. (2000) Dual roles for c-Jun N-terminal kinase in developmental and stress responses in cerebellar granule neurons. J. Neurosci. 20, $7602-7613$.

Coffey E. T., Smiciene G., Hongisto V., Cao J., Brecht S., Herdegen T. and Courtney M. J. (2002) c-Jun N-terminal protein kinase (JNK) $2 / 3$ is specifically activated by stress, mediating c-Jun activation, in the presence of constitutive JNK1 activity in cerebellar neurons. J. Neurosci. 22, 4335-4345.

Estus S., Zaks W. J., Freeman R. S., Gruda M., Bravo R. and Johnson E. M. Jr (1994) Altered gene expression in neurons during programmed cell death: identification of c-jun as necessary for neuronal apoptosis. J. Cell Biol. 127, 1717-1727.

Glicksman M. A., Chiu A. Y., Dionne C. A. et al. (1998) CEP-1347/ KT7515 prevents motor neuronal programmed cell death and injury-induced dedifferentiation in vivo. J. Neurobiol. 35, 361-370.

Goldschmidt R. B. and Steward O. (1980) Preferential neurotoxicity of colchicine for granule cells of the dentate gyrus of the adult rat. Proc. Natl Acad. Sci. USA 77, 3047-3051.

Goldschmidt R. B. and Steward O. (1982) Neurotoxic effects of colchicine: differential susceptibility of CNS neuronal populations. Neuroscience 7, 695-714.

Ham J., Babij C., Whitfield J., Pfarr C. M., Lallemand D., Yaniv M. and Rubin L. L. (1995) A c-Jun dominant negative mutant protects sympathetic neurons against programmed cell death. Neuron 14, 927-939.

Ham J., Eilers A., Whitfield J., Neame S. J. and Shah B. (2000) c-Jun and the transcriptional control of neuronal apoptosis. Biochem. Pharmacol. 60, 1015-1021.

Hanson M. and Edstrom A. (1978) Mitosis inhibitors and axonal transport. Int. Rev. Cytol. Suppl. 7, 373-402.

Harper S. J. and LoGrasso P. (2001) Signalling for survival and death in neurones: the role of stress-activated kinases, JNK and p38. Cell. Signal. 13, 299-310.

Harris C. A. and Johnson E. M. Jr (2001) BH3-only Bcl-2 family members are coordinately regulated by the JNK pathway and require Bax to induce apoptosis in neurons. J. Biol. Chem. 276, 37 754-37 760.

Harris C. A., Deshmukh M., Tsui-Pierchala B., Maroney A. C. and Johnson E. M. Jr (2002) Inhibition of the c-Jun N-terminal kinase signaling pathway by the mixed lineage kinase inhibitor CEP-1347 (KT7515) preserves metabolism and growth of trophic factordeprived neurons. J. Neurosci. 22, 103-113.

Herdegen T., Skene P. and Bähr M. (1997) The c-Jun transcription factor - bipotential mediator of neuronal death, survival and regeneration. Trends Neurosci. 20, 227-231.

Ho L., Osaka H., Aisen P. S. and Pasinetti G. M. (1998) Induction of cyclooxygenase (COX)-2 but not COX-1 gene expression in apoptotic cell death. J. Neuroimmunol. 89, 142-149.

Kallunki T., Su B., Tsigelny I., Sluss H. K., Derijard B., Moore G., Davis R. and Karin M. (1994) JNK2 contains a specificity-determining region responsible for efficient c-Jun binding and phosphorylation. Genes Dev. 8, 2996-3007.

Kuan C. Y., Whitmarsh A. J., Yang D. D. et al. (2003) A critical role of neural-specific JNK3 for ischemic apoptosis. Proc. Natl Acad. Sci. USA 100, 15 184-15 189.
Leist M., Volbracht C., Kuhnle S., Fava E., Ferrando-May E. and Nicotera P. (1997) Caspase-mediated apoptosis in neuronal excitotoxicity triggered by nitric oxide. Mol. Med. 3, 750-764.

Le-Niculescu H., Bonfoco E., Kasuya Y., Claret F. X., Green D. R. and Karin M. (1999) Withdrawal of survival factors results in activation of the JNK pathway in neuronal cells leading to Fas ligand induction and cell death. Mol. Cell. Biol. 19, 751-763.

Liu Z., Gastard M., Verina T., Bora S., Mouton P. R. and Koliatsos V. E. (2001) Estrogens modulate experimentally induced apoptosis of granule cells in the adult hippocampus. J. Comp. Neurol. 441, $1-8$.

Mandelkow E. M., Stamer K., Vogel R., Thies E. and Mandelkow E. (2003) Clogging of axons by tau, inhibition of axonal traffic and starvation of synapses. Neurobiol. Aging 24, 1079-1085.

Maroney A. C., Finn J. P., Bozyczko-Coyne D., O'Kane T. M., Neff N. T., Tolkovsky A. M., Park D. S., Yan C. Y., Troy C. M. and Greene L. A. (1999) CEP-1347 (KT7515), an inhibitor of JNK activation, rescues sympathetic neurons and neuronally differentiated PC12 cells from death evoked by three distinct insults. J. Neurochem. 73, 1901-1912.

Maroney A. C., Finn J. P., Connors T. J. et al. (2001) Cep-1347 (KT7515), a semisynthetic inhibitor of the mixed lineage kinase family. J. Biol. Chem. 276, 25 302-25 308.

Matsuoka Y., Okazaki M., Zhao H., Asai S., Ishikawa K. and Kitamura Y. (1999) Phosphorylation of c-Jun and its localization with heme oxygenase-1 and cyclooxygenase-2 in CA1 pyramidal neurons after transient forebrain ischemia. J. Cereb. Blood Flow Metab. 19, $1247-1255$.

Mielke K. and Herdegen T. (2000) JNK and p38 stresskinases degenerative effectors of signal transduction-cascades in the nervous system. Prog. Neurobiol. 61, 45-60.

Minden A., Lin A., Smeal T., Derijard B., Cobb M., Davis R. and Karin M. (1994) c-Jun N-terminal phosphorylation correlates with activation of the JNK subgroup but not the ERK subgroup of mitogenactivated protein kinases. Mol. Cell. Biol. 14, 6683-6688.

Nath R., Raser K. J., Stafford D., Hajimohammadreza I., Posner A., Allen H., Talanian R. V., Yuen P., Gilbertsen R. B. and Wang K. K. (1996) Non-erythroid alpha-spectrin breakdown by calpain and interleukin 1 beta-converting-enzyme-like protease(s) in apoptotic cells: contributory roles of both protease families in neuronal apoptosis. Biochem. J. 319, 683-690.

Pei J. J., Braak E., Braak H., Grundke-Iqbal I., Iqbal K., Winblad B. and Cowburn R. F. (2001) Localization of active forms of C-jun kinase (JNK) and p38 kinase in Alzheimer's disease brains at different stages of neurofibrillary degeneration. J. Alzheimers Dis. 3, 41-48.

Pozas E., Aguado F. and Ferrer I. (1999) Localization and expression of Jun-like immunoreactivity in apoptotic neurons induced by colchicine administration in vivo and in vitro depends on the antisera used. Acta Neuropathol. 98, 119-128.

Saporito M. S., Brown E. M., Miller M. S. and Carswell S. (1999) CEP1347/KT-7515, an inhibitor of c-jun N-terminal kinase activation, attenuates the 1-methyl-4-phenyl tetrahydropyridine-mediated loss of nigrostriatal dopaminergic neurons In vivo. J. Pharmacol. Exp. Ther. 288, 421-427.

Savage M. J., Lin Y. G., Ciallella J. R., Flood D. G. and Scott R. W. (2002) Activation of c-Jun N-terminal kinase and p38 in an Alzheimer's disease model is associated with amyloid deposition. J. Neurosci. 22, 3376-3385.

Shoji M., Iwakami N., Takeuchi S., Waragai M., Suzuki M., Kanazawa I., Lippa C. F., Ono S. and Okazawa H. (2000) JNK activation is associated with intracellular beta-amyloid accumulation. Brain Res. Mol. Brain Res. 85, 221-233.

Spillantini M. G. and Goedert M. (1998) Tau protein pathology in neurodegenerative diseases. Trends Neurosci. 21, 428-433. 
Troy C. M., Rabacchi S. A., Xu Z., Maroney A. C., Connors T. J., Shelanski M. L. and Greene L. A. (2001) beta-Amyloid-induced neuronal apoptosis requires c-Jun N-terminal kinase activation. J. Neurochem. 77, 157-164.

Vaudano E., Rosenblad C. and Björklund A. (2001) Injury induced c-Jun expression and phosphorylation in the dopaminergic nigral neurons of the rat: correlation with neuronal death and modulation by glialcell-line-derived neurotrophic factor. Eur. J. Neurosci. 13, 1-14.

Volbracht C., Leist M. and Nicotera P. (1999) ATP controls neuronal apoptosis triggered by microtubule breakdown or potassium deprivation. Mol. Med. 5, 477-489.

Volbracht C., Leist M., Kolb S. A. and Nicotera P. (2001) Apoptosis in caspase-inhibited neurons. Mol. Med. 7, 36-48.

Wang L. H., Besirli C. G. and Johnson E. M. Jr (2004) Mixed-lineage kinases: a target for the prevention of neurodegeneration. Annu. Rev. Pharmacol. Toxicol. 44, 451-474.

West M. J. and Gundersen H. J. (1990) Unbiased stereological estimation of the number of neurons in the human hippocampus. J. Comp. Neurol. 296, 1-22.
West M. J., Slomianka L. and Gundersen H. J. (1991) Unbiased stereological estimation of the total number of neurons in thesubdivisions of the rat hippocampus using the optical fractionator. Anat. Rec. 231, 482-497.

Whitfield J., Neame S. J., Paquet L., Bernard O. and Ham J. (2001) Dominant-negative c-Jun promotes neuronal survival by reducing BIM expression and inhibiting mitochondrial cytochrome $c$ release. Neuron 29, 629-643.

Xu Z., Maroney A. C., Dobrzanski P., Kukekov N. V. and Greene L. A. (2001) The MLK family mediates c-Jun N-terminal kinase activation in neuronal apoptosis. Mol. Cell. Biol. 21, 47134724.

Zhu X., Raina A. K., Rottkamp C. A., Aliev G., Perry G., Boux H. and Smith M. A. (2001) Activation and redistribution of c-jun Nterminal kinase/stress activated protein kinase in degenerating neurons in Alzheimer's disease. J. Neurochem. 76, 435-441. 